\title{
Patients with Idiopathic Inflammatory Myopathies and Primary involvement of Heart Muscle and Cardiovascular Complications
}

\author{
Aleksandra Opinc ${ }^{1}$, Marcin Makowski ${ }^{2}$ and Joanna Makowska*1 \\ ${ }^{1}$ Department of Rheumatology, Medical University of Lodz, Poland \\ ${ }^{2}$ Department of Intensive Care in Cardiology, Medical University of Lodz, Poland
}

Received: May 28, 2018; Published: June 08, 2018

*Corresponding author: Joanna Makowska, Department of Rheumatology, Medical University of Lodz, Pieniny 30, 90-003 Lodz, Poland

\begin{abstract}
Idiopathic inflammatory myopathies are rare connective tissue disorders, in which inflammatory process affects mainly skeletal muscles leading to their progressive weakness. However internal organs can be affected as well, deteriorating the course of disease. We present cases of two patients with cardiac manifestations as predominant symptoms of idiopathic inflammatory myopathies. In both cases arrhythmia was the leading symptom preceding diagnosis of myositis.
\end{abstract}

Keywords: Myositis; Myopathy; Arrhythmia

Abbreviations: IIM: Idiopathic Inflammatory Myopathy; PM: Polymyositis; DM: Dermatomyositis; IBM: Inclusion Body Myositis; MRI: Magnetic Resonance Imaging; CRP: C-Reactive Protein; CK: Creatine kinase; CK-MB: Creatine kinase Muscle-Brain isoenzyme; DDD pacemaker: Dual Chamber pacemaker; CRT-P: Cardiac Resynchronization Therapy Pacemaker; LV: Left ventricle; LVEF: Left Ventricle Ejection Fraction; LDH: Lactate Dehydrogenase; ANA: Antinuclear antibodies; ESR: Erythrocyte Sedimentation Rate; pANCA: Perinuclear Anti-Neutrophil Cytoplasmic Antibodies; ECG: Electrocardiography; CHF: Congestive Heart Failure

\section{Background}

Idiopathic inflammatory myopathies (IIM), including polymyositis (PM), dermatomyositis (DM), inclusion body myositis (IBM), juvenile myositis, necrotizing myositis and myositis in course of overlapping syndrome is a heterogeneous group of connective tissue diseases. Muscle-specific antibodies are detected in sera of $50-70 \%$ patients, what suggests the role of autoimmunity in the pathogenesis [1]. Most recognized symptom of IIM is progressive symmetric muscle weakness, affecting predominantly proximal muscles of upper and lower extremities. Approximately 1 in 3 patients suffers from myalgia and muscle tenderness [1]. In dermatomyositis muscle symptoms are accompanied by various cutaneous lesions (i.e. heliotrope rash and Gottron's papules). However, general symptoms and internal organ involvement are reported in idiopathic inflammatory myopathies as well and these symptoms can sometimes precede the muscular complaints [1,2]. Organ involvement in IIM could significantly alter both the course of the disease and prognosis for the patients. Non-specific course of the disease frequently leads to delayed or incorrect diagnosis. We present two patients with IIM in whom severe cardiac involvement was the first manifestation of the disease.

\section{CASE A}

Patient A, a 67-year old female with past medical history of autoimmune hepatitis was referred to the Department of Rheumatology with suspicion of idiopathic myopathy. The first symptoms started in 2011 when patient was referred to cardiology unit due to palpitations. The diagnosis of sick sinus syndrome and tachy-brady syndrome was stated (focal atrial contraction, paroxysmal atrial tachycardia and sinus bradycardia). In April 2012 she experienced chest pain with elevated troponin $\mathrm{T}$ level and due to suspected acute coronary syndrome coronary angiography was performed but did not reveal atherosclerotic plaques. Echocardiography revealed global hypokinesis with ejection fraction $50 \%$, moderate mitral and tricuspid insufficiency and enlargement of all chambers.

Magnetic resonance of the heart confirmed global hypokinesis with late enhancement localized subendocardial in the area of the inferior wall and inferior part of the lateral wall at the basal and middle segments up to $60-70 \%$ of the wall. Traced linear enhancements within the interventricular septum and global systolic dysfunction indicated possible cardiac involvement as a consequence 
of inflammatory process. Because of accompanying complaints such as muscle tenderness and weakness of proximal muscles of lower extremities as well as elevated levels of creatine kinase (up to $3962 \mathrm{U} / \mathrm{l}$ ), myoglobin ( $\mu \mathrm{g}$ to $633,9 \mu \mathrm{g} / \mathrm{l}$ ) and CRP (6,1 mg/l) patient was referred to Rheumatology Department. On admission patient presented muscle weakness of shoulder and pelvic girdle and cutaneous symptoms (heliotrope rash and Gottron's papules).

Antinuclear antibodies (titre 1:640), anti-Mi-2 and anti-Ro-52 autoantibodies were detected. In electromyography she had features of primary muscle involvement. Based on clinical symptoms and additional tests the diagnosis of idiopathic inflammatory myopathy (dermatomyositis) was posed and treatment with oral corticosteroids $(1 \mathrm{mg} / \mathrm{kg})$ with tapering of the dose and azathioprine was started, what led to improvement of muscle strength and disappearing of the cutaneous lesions. Muscle enzymes (CK, CK-MB, troponin, myoglobin) decreased significantly and in 2013 returned to normal values after patient was treated with $4 \mathrm{mg}$ methylprednisolone and $150 \mathrm{mg}$ of azathioprine for 1,5 year. Due to recurrent syncope (sinus bradycardia, pauses up to 10 seconds) patient required urgent pacemaker implantation. Dual chamber pacemaker was done in January 2013. For 3 years the patient was mainly under supervision of cardiologists.

In January 2016 she experienced dyspnoea, palpitations, deterioration of physical exercise tolerance and signs of heart failure. Echocardiography examination revealed reduced ejection fraction, which has deteriorated over time from $50 \%$ to $30 \%$. The heart muscle enzymes were within normal limits and muscle enzymes as well as inflammatory markers were elevated (CK 1092U/l, CRP $7,2 \mathrm{mg} / \mathrm{l}$ ). Due to atrial electrode dysfunction (high threshold and undersensing), heart failure progression and reduced ejection fraction she was scheduled for up-grade DDD pacemaker to resynchronisation therapy with removal of dysfunctional atrial electrode. In July 2016 electroanatomic mapping of right atrium and coronary sinus was performed. Low-voltage area covering the whole atrium was detected. New atrial electrode was implanted near coronary sinus ostium according to proper electrophysiology parameters. Due to reduced ejection fraction dual chamber pacemaker was upgraded for CRT-P. Although there was a transient improvement in ejection fraction after CRT-P implantation, patient's condition has deteriorated over time (last ejection fraction 26\%).

Patient was hospitalized twice in 2016 in the Department of Rheumatology, during first hospitalization in February patient received 3 pulses of methylprednisolone due to deterioration of muscle strength and elevation of muscle enzymes (CK $800 \mathrm{U} / \mathrm{l}$ ), with initial improvement (decrease of CK values to $356 \mathrm{U} / \mathrm{l}$ ). She was re-hospitalized in Rheumatology Department in November 2016 due to progression of heart failure, the pharmacological treatment was modified (increasing diuretics dose) and the dose of glucocorticosteroids was increased to $12 \mathrm{mg}$ methylprednisone with disappearance of ankle oedema and dyspnoea. In June 2017 cardiac involvement resulted in fatal outcome, leading to death in the unknown mechanism of cardiac arrest.

\section{CASE B}

Patient B is a male, aged 67, who was referred to the Department of Rheumatology from the Cardiology Department where he was hospitalized due to atrial flutter and elevated troponin levels (636 ng/L at admission to Cardiology Department) without chest pain. As the levels of troponin remained stable (between 630-752 ng/L within 7 days, with no upward trend) and echocardiography examination suggested tachy-cardiomiopathy coronary angiography was not performed. Patient was diagnosed with persistent atrial flutter (successfully terminated) and left bundle branch block. MRI was performed and displayed foci of late enhancement localised subepicardial in the basal area of the intraventricular septum and lateral wall, what indicated inflammatory process. Features of an active inflammation, such as myocardial oedema, were not reported. Global LV systolic function was reduced with the presence of segmental contractility dysfunction and ejection fraction of $36 \%$.

After the heart rate decreased, echocardiography revealed LVEF of $46 \%$ and a trace of pericardial effusion. As the levels of muscle enzymes were elevated (CK 1091-1105 U/l, CK-MB mass 146,3$183,9 \mathrm{ng} / \mathrm{ml}$, myoglobin 419,80 $\mathrm{gg} / \mathrm{l}$, LDH 504U/l) and antinuclear antibodies were detected (ANA 1:2560) patient was transferred to the Department of Rheumatology with the suspicion of idiopathic inflammatory myopathy. On admission to the Department of Rheumatology he complained of progressive weakness of muscles of pelvic and shoulder girdle, fatigue, impaired tolerance of physical exercise accompanied with morning stiffness. In past history patient reported that he was hospitalized several times on pulmonology due to subfebril states, dyspnoea and crepitation where the diagnosis of interstitial pneumonia was posed in 2011 (confirmed in high resolution computed tomography-ground glass opacities). Family history included systemic lupus erythematous in patient's mother. The physical examination on admission revealed impaired muscle strength and ulcerations on the top of the fingers. In laboratory tests, muscle enzymes were elevated (CK 948 U/l, myoglobin 377,3 $\mu \mathrm{g} / \mathrm{L}, \mathrm{LDH} 499,9 \mathrm{U} / \mathrm{L}$ ), CRP and ESR were within normal limits.

In immunological tests antinuclear antibodies (ANA 1:640) with anti-Ro52, anti-PM/Scle100, anti-PM/Scle75, also pANCA antibodies were detected. Other causes of myopathies (toxic, endocrinological) were excluded. Muscle biopsy and electromyography confirmed the diagnosis of idiopathic inflammatory myopathy (scleromyositis). Treatment with oral corticosteroids and azathioprine was implemented and led to decline in $\mathrm{CK}$ and myoglobin levels. One month after hospitalization patient was admitted to cardiology unit where electric cardioversion was performed and sinus rhythm was restored. Ejection fraction improved from $46 \%$ to $60 \%$ over time after diagnosis. Currently, majority of clinical symptoms including muscle weakness, dyspnoea and palpitations disappeared, however supraventricular ectopic beats are still observed in $24 \mathrm{~h}$ ECG monitoring (latest examination performed in March 2017 revealed 10386 supraventricular premature beats) but recurrence of atrial flutter was not observed. Muscle enzymes and acute phase proteins remain within normal limits. 


\section{Discussion}

Cardiac involvement in idiopathic inflammatory myopathy was firstly reported by Oppenheim in 1899 [3,4]. Recently, numerous data indicates that prevalence of cardiac complications in this group of patients is more frequent than it was previously expected, however data vary significantly depending on the study [4]. According to literature, cardiac involvement in course of IIM usually remains subclinical [4]. Most frequently observed disturbances include arrhythmia or conduction disorders, faster development of atherosclerosis and its complications (like coronary artery disease and myocardial infarctions) however cardiomyopathy, myocarditis, and pulmonary heart disease are also observed [2-4]. Abnormalities in ECG are frequently observed in IIM patients with the prevalence of these disorders ranging from $23,1 \%$ even to $81,81 \%[5,6]$. Frequently observed abnormalities include non-specific changes of ST-T segment and T-wave morphology [4,7].

Conduction disorders in the form of atrioventricular blocks of all grades and Hiss bundle branch blocks are also frequently diagnosed in PM/DM patients with the prevalence of 25-38,5\% [4]. Symptomatic involvement of cardiac muscle usually manifests as congestive heart failure (CHF) [4]. The prevalence of CHF in patients with IIM was 3-45\% according to older studies, yet in recent metaanalysis of 26 publicaations this complication affected up to $77 \%$ of the studied population. [4,8]. Our cases show that cardiac symptoms can precede or appear at the same time as peripheral myopathy and symptoms like dyspnoea and palpitations being the result of the arrhythmia and heart failure can be the dominant manifestation of the disease. Myocardial lesions could be immense, as it was described in a case of the PM patient requiring myocardial transplant due to dilated cardiomyopathy [9]. Inflammatory induced fibrosis should be considered as causative factor for cardiac complications, as it was reported in a case of a man, in whom the post-mortem study revealed coexistence of atherosclerotic foci with fibrosis of all of the heart chambers and intraventricular septum [10].

Our presented both conduction abnomrmalities and arrhythmia. Also according to the literature, patients with IIM may require pacemaker implantation, what illustrates the potential threat to the cardiac conductive system $[10,11]$. It remains unclear whether cardiac involvement correlates with intensity of skeletal muscle inflammation $[4,12,13]$. Myocardial involvement was proven to be an unfavourable prognostic factor $[11,14,15]$. A poorer 8-year survival was observed in patients with cardiac lesions in course of PM / DM when compared to patients without myocardial involvement [15]. In addition, cardiovascular diseases frequently result in fatal outcome [6,14-16]. Presented cases emphasize the need for interdisciplinary cooperation between medical professionals of different specialities such as rheumatologists, cardiologists and pulmonologists, especially if the patient presents an unobvious course of the disease.

\section{Conclusion}

Connective tissue disorders including idiopathic inflammatory myopathies should be considered as a possible diagnosis in pa- tients presenting cardiac symptoms and concomitant non-specific musculoskeletal or cutaneous symptoms. Due to the high frequency of subclinical course, cardiovascular assessment in this group of patients seems to be necessary and justified, even in asymptomatic patients. Widely available, non-invasive tests assessing morphology and function of the cardiovascular system, such as electrocardiogram or echocardiography, allow to detect abnormalities at an early stage, and thus prevent or slow down further progression. In patients with diagnosis of acute coronary syndrome with no changes in coronary arteries and elevated troponin levels, inflammatory myopathies should be considered as one of differential diagnosis.

\section{References}

1. Marvi U, Chung L, Fiorentino DF (2012) Clinical Presentation and Evaluation of Dermatomyositis. Indian J Dermatol 57(5): 375-381.

2. Findlay AR, Goyal NA, Mozaffar T (2015) An overview of polymyositis and dermatomyositis. Muscle Nerve 51(5): 638-656.

3. Gupta R, Wayangankar SA, Targoff IN, Hennebry TA (2011) Clinical cardiac involvement in idiopathic inflammatory myopathies: a systematic review. Int J Cardiol 148(3): 261-270.

4. Zhang L, Wang GC, Ma L, Zu N (2012) Cardiac involvement in adult polymyositis or dermatomyositis: a systematic review. Clin Cardiol 35(11): 686-691.

5. Hebert CA, Byrnes TJ, Baethge BA, Wolf RE, Kinasewitz GT (1990) Exercise limitation in patients with polymyositis. Chest 98(2): 352-357.

6. Bohan A, Peter JB, Bowman RL, Pearson CM (1977) Computer-assisted analysis of 153 patients with polymyositis and dermatomyositis. Medicine (Baltimore) 56(4): 255-286.

7. Byrnes TJ, Baethge BA, Wolf RE (1991) Noninvasive cardiovascular studies in patients with inflammatory myopathy. Angiology 42(10): 843-848.

8. Lundberg IE (2005) Cardiac involvement in autoimmune myositis and mixed connective tissue disease. Lupus 14(9): 708-712.

9. Afzal A, Higgins RS, Philbin EF (1999) Heart transplant for dilated cardiomyopathy associated with polymyositis. Heart 82(4): e4.

10. Lightfoot PR, Bharati S, Lev M (1977) Chronic dermatomyositis with intermittent trifascicular block. An electrophysiologic-conduction system correlation. Chest 71(3): 413-416.

11. Oka M, Raasakka T (1978) Cardiac involvement in polymyositis. Scand J Rheumatol 7(4): 203-208.

12. Lundberg IE (2006) The heart in dermatomyositis and polymyositis. Rheumatology (Oxford) 45 (Suppl 4): iv18-21.

13. Sharratt GP, Danta G, Carson PH (1977) Cardiac abnormality in polymyositis. Ann Rheum Dis 36(6): 575-578.

14. Dankó K, Ponyi A, Constantin T, Borgulya G, Szegedi G (2004) Longterm survival of patients with idiopathic inflammatory myopathies according to clinical features: a longitudinal study of 162 cases. Medicine (Baltimore) 83(1): 35-42.

15. Marie I (2012) Morbidity and mortality in adult polymyositis and dermatomyositis. Curr Rheumatol Rep 14(3): 275-285.

16. Xiao Y, Zuo X, You Y, Luo H, Duan L, et al. (2016) Investigation into the cause of mortality in 49 cases of idiopathic inflammatory myopathy: A single center study. Exp Ther Med 11(3): 885-889. 
(C) This work is licensed under Creative

Submission Link: https://biomedres.us/submit-manuscript.php

Assets of Publishing with us
RESEARCHES $\quad$\begin{tabular}{l} 
Global archiving of articles \\
- Immediate, unrestricted online access \\
\hline
\end{tabular}

